\title{
A Brief Guide for the Continuously Evolving $\mu$ Controller Raspberry PI Mod.B
}

\author{
M. Papoutsidakis \\ Dept. of Automation \\ Engineering \\ PUAS, Athens, Greece
}

\author{
A. Chatzopoulos \\ Dept. of Automation \\ Engineering \\ PUAS, Athens, Greece
}

\author{
K. Kalovrektis \\ Dept. of Informatics \\ University of Thessaly \\ Lamia, Greece
}

\author{
C. Drosos \\ Dept. of Automation \\ Engineering \\ PUAS, Athens, Greece
}

\begin{abstract}
It is an accepted fact that, as the years go by, technology rapidly evolves. For this reason, it is absolutely necessary to constantly watch its evolution, to search for new ways for its progression, adjusting to the new conditions and demands. This effort can be mainly fulfilled by computers. Computers allow us to comprehend the development of technology, assisted by the growing development of the tool-programs that can be used. Another fundamental stepping stone for the technological evolution is the electrical machines which replace manual labor, since they are faster, more precise, and dependable. On a long term, using the electrical machines decreases the cost, whereas manual labor loses its prestige as the years go by. The present project extensively discusses the Raspberry Pi, one of the most sophisticated pocket computer models, which is able to conduct more complex implementations than the rest of the available models, using more program languages.
\end{abstract}

\section{Keywords}

Raspberry, microcontroller

\section{INTRODUCTION}

In the above abstract, there is an attempt to describe what is to follow in the rest of the paper by indicating the main issues referring to Respberry microcontroller. It must also be stated that this research work reflects on a bigger project which was ans still is undertaken in Intelligent Systems Laboratory, Automation Engineering Dept, PUAS. Therefore in case we refer to "chapters" throughout this paper, the reader can get all respective information by sending email to the corresponding author.

\section{DESCRIPTION AND APPLICATIONS}

In the $1^{\text {st }}$ chapter, we will make a small introduction about the Raspberry $\mathrm{Pi}$, mentioning its definition and its history, referring to the date of the release of each model and some details about each one of them. We will, also, discuss the differentiations of each model and, this very chapter will make us understand what we can do with each one of the models.

In the $2^{\text {nd }}$ chapter, we will refer to the raspberry hardware. First of all, the pins of the Raspberry Pi will be introduced and analyzed. The attachments needed for the activation of the Raspberry Pi will be mentioned and the right steps for the installation of the NOOBS operating system to the SD Card will be studied. Moreover, the RASPBIAN operating system will be installed to the Raspberry Pi 3. Finally, the first steps that we need to make once the Raspberry Pi turns on will be mentioned.

In the $3^{\text {rd }}$ chapter we will talk about the SSH. We will closely take a look at the steps needed in order to install the
RASPBIAN operating system to the SD Card. We will see what the SSH is, as well.

In the $4^{\text {th }}$ chapter, we will study the examples where the micro-controller is used, the attachments needed for each project, and we will analyze the circuits for each of the examples. We will be citing the circuit through the FRITZING and we will be discussing the code of each example so that the application can function. The examples will be of a gradual difficulty.

The present work aims to inform readers about an innovative micro-controller; the Raspberry Pi 3 Model B. The truth is that only few people are aware of this micro-controller. For this reason, our goal is to make the micro-controller more comprehensive to everybody.

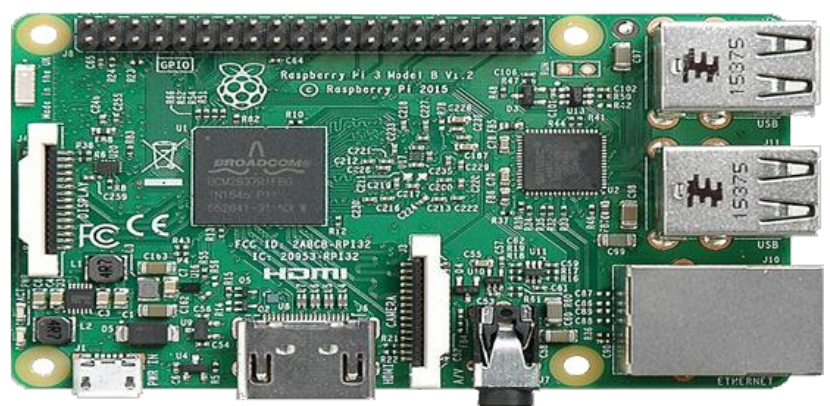

Fig.1 : The microcontroller board

To start with, the $\mathbf{1}^{\text {st }}$ chapter will introduce us to the basic information of the Raspberry Pi. The Raspberry Pi is a smallsized pocket computer of low cost, which has been available in the United Kingdom since 2012. The aim of its inspirers, Eben Upton, Rob Mullins, Jack Lang and Alan Mycroft, was to draw the school students' attention to coding. The first models that came out were the Raspberry Pi Model A, the Raspberry Pi Model A+ and the Raspberry Pi Model B. These models were followed by the Raspberry Pi Model B+. In February 2015, the Raspberry Pi 2 Model B came out followed by the Raspberry Pi Zero in November 2015. In February 2016 the latest Raspberry Pi was launched, the Raspberry Pi 3 Model B. The developments of the technology generally, as well as the development of the micro-controller models have increased the capabilities of each model. In relation to the first models, the Pins have been increased from 26 to 40 and the USB ports from 2 to 4 . The capacity of RAM has also, been increased. We use the Raspberry Pi over the rest of the micro-controller due to its small size, low cost and its easy internet and Linux access. It also, enables us to program in many languages. However, the Raspberry Pi apart from the advantages already mentioned, has a few disadvantages, allowing us to use other micro-controllers. One disadvantage is that the access to the material does not happen in real time. Other disadvantages are the low power of the 
inductive loads, the absence of an embedded converter which converts the analog load to a digital one, and the closed circuit design of the material. In our everyday life, we use the Raspberry $\mathrm{Pi}$ in order to make a computer by connecting a keyboard and a computer mouse. This computer enables us to watch movies on television, since the Raspberry can be used as a Media Center Pc. In addition, we can have a machine which simulates old games by connecting a portable hard disk to the Raspberry. We can also, have a system which downloads files by connecting a portable hard disk to the Raspberry, since it consumes less power. Furthermore, we have the ability to create our own cloud service so that we access our files through the internet without using the familiar to all methods (e.g. Yahoo, Dropbox).

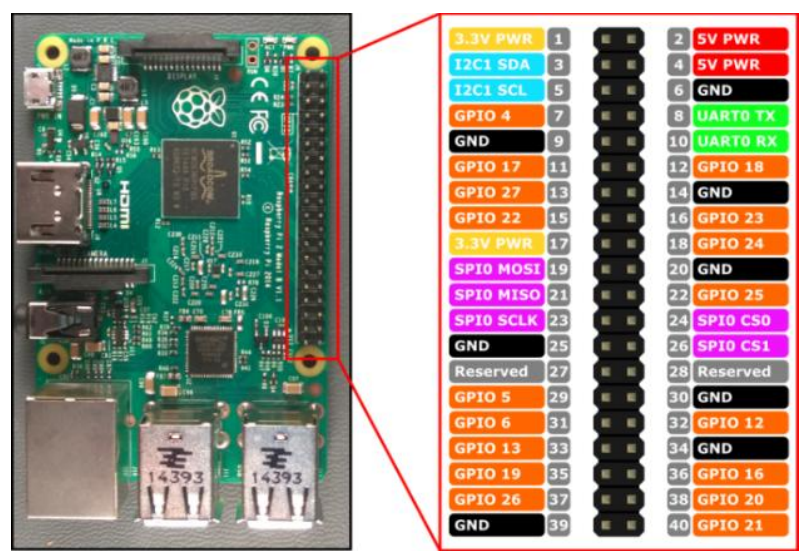

Fig.2 : The microcontroller port description

Chapter 2 discusses the hardware of the Raspberry Pi. Firstly, the pins of the micro-controller are analyzed. We will learn how the numeration of each pin is done, how they are called and the category each pin falls into, which will conclude to the analysis of these categories. These are the Pins which supply voltage, the Pins which are grounds, the ones for general use, the ones which fall under the category of the SPI, PWM, $\mathrm{I}^{2} \mathrm{C}$, UART and CLK and the Pins which are not used. Then the attachments needed for the Raspberry Pi to function and be turned on are analyzed. These attachments are the feed, the Micro SD Card and the adapter. The steps needed to install the NOOBS operating system on the SD Card is also, analyzed in this chapter. An important step is to download a program that is suggested by the NOOBS. This program allows us to format the SD Card. Once the format is over, we have to visit a link in order to download the latest NOOBS edition. We can download the file in two ways, but we choose the NOOBS LITE edition to save space. So, following all the right steps leads to the installation of the NOOBS in the SD Card. Furthermore, this chapter is referred to the steps for the installation of the RASPBIAN to the Raspberry Pi. We will see the way in which the attachments are laded to the Raspberry Pi and the holes. These attachments are the Micro SD Card, the case of the Raspberry Pi, the HDMI and the Ethernet wires, the mouse and the keyboard. At the end of the chapter, the steps needed for the first startup of the Raspberry $\mathrm{Pi}$ are mentioned too. Having correctly fulfilled all the steps we will ready to enter the world of the Raspberry Pi and start experimenting.

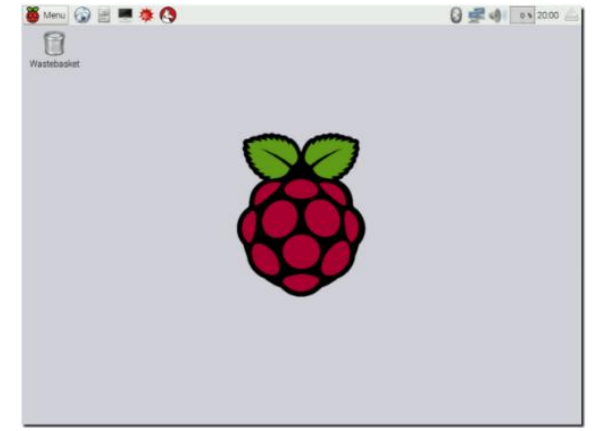

Fig.3: The microcontroller main interface

Chapter 3 refers to the SSH. First of all, we will see how the installation of the RASPBIAN operating system to the SD Card is done. In order for this installation to happen, we must enter a website where there are two choices for the installation. One of them is the regular edition of the RASPBIAN and the other one is the LITE edition. We use the LITE edition due to its smaller size. Also, the RASPBIAN can be downloaded through torrent apart from the website. Next, another program the WIN 32 DISK is necessary to be used. This program which can be downloaded for free allows us to code the image file of the operating system on the SD Card. Another necessary program also free of charge, is the ADVANCED IP SCANNER. This will help us find the IP address of the Raspberry Pi 3 Model B, which will connected to the router with an Ethernet wire, as well as the IP address of every other device connected to our domestic network. A third free of cost program to access the command line of the Raspberry Pi is PuTTY. PuTTY is a remote control program which operates with the SSH protocol. All the steps must be correctly fulfilled, as well, so that the login of Raspberry opens at the end. This way we will be able to put the common username (pi) and password (raspberry) so that we access the Raspberry Pi command line.

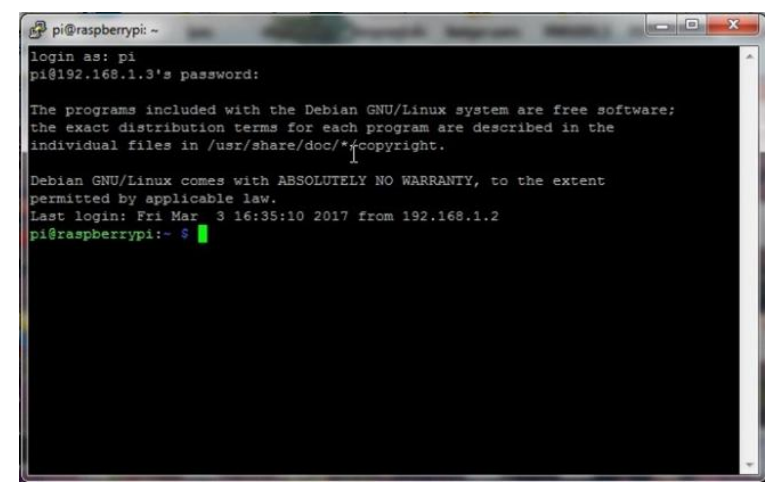

Fig.3: The microcontroller assembly window

In chapter 4 we will see simple examples of how one can use the Raspberry Pi 3 Model B micro-controller. The difficulty of the examples will be gradual. At first, we are going to see a simple example and the further we go the more difficult the examples will be. This chapter includes six examples. The examples are:

A Led which turns on when voltage is supplied to it.

A Led which turns on by pushing a Button.

A Led, the brightness of which I adjusted by a Potentiometer.

The activation of a DC Motor for a rightward and a leftward 
motion.

The detection of the distance in the Raspberry with the HCSR04.

The activation of a servo-motor with the Raspberry Pi by supplying voltage from butteries.

For each example, the attachments needed for the actualization of each project will be scrutinized. Most of the times, the attachments used will be the same as in the previous projects. However, new attachments will often be used. All of the attachments will be, of course analyzed either we come across them numerous times or in individual projects. The attachments needed for all of the examples are: resistors (the value of each resistor will change depending on the project), led, breadboard (which will be used in every project), the Raspberry Pi 3 Model B (which will also be used in every project, a button, a potentiometer, an MCP3008 (which will be seen in the $3^{\text {rd }}$ example, meaning the adjustment of the led brightness by a potentiometer), butteries , a DC Motor, an L293D (which will be seen in the $4^{\text {th }}$ example, more specifically, in the activation of the DC Motor for a rightward and a leftward motion), an HC-SR04 sonar, a servo-motor and finally, the wires for the connections, Then, we will analyze the connection of the electrical circuit for each project. This connection will be used in order to simulate the real connection through Fritzing. Having the fritzing circuit assures us that we have not made any mistake during the real (including attachments) connection. Fritzing will give us the ability to become acquainted with the electrical design, as soon as we simulate the circuit. After the completion of all these, we will be ready to program. The corresponding code for each example will be cited. Also, every point of the code will be explained command by command. In the $1^{\text {st }}$ example we will study the following attachments: a Raspberry Pi 3 Model B, a Breadboard, a red led a $330 \Omega$ resistor and the wires for the connection. Furthermore, we will see the circuit that needs to be implemented, the circuit through fritzing, the electrical design and the code. We will explain how each connection of the attachments is done, and talk about which Raspberry Pi pins the wires are attached to.

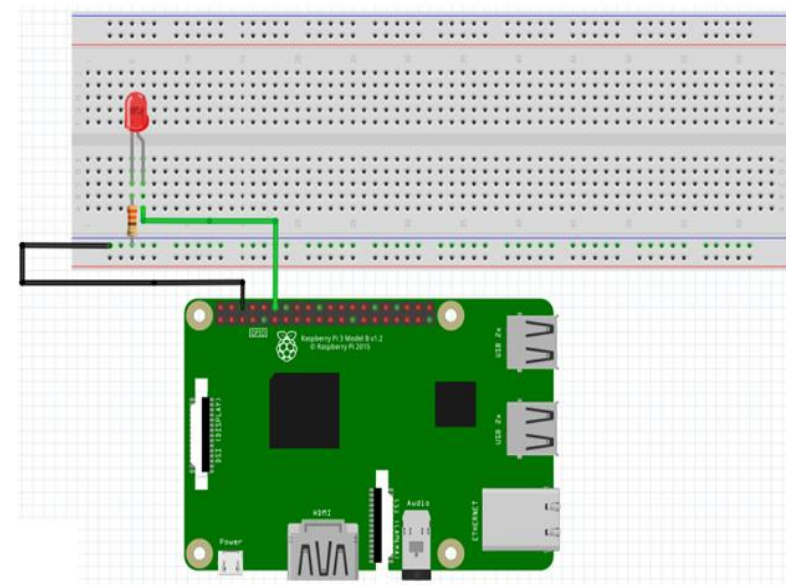

Fig.4: The first application wirings

In the $2^{\text {nd }}$ example, the attachments that will be studied are about the same as before (a Raspberry Pi 3 Model B, a Breadboard, a Led, a $220 \Omega$ resistor and wires for the connection), but, also, one more (which has not been used so far), a Button. So, we will see the function of the Button, the circuit that needs to be implemented, the circuit through the fritzing program and, the electrical design of fritzing and, last but not least, the code needed for the activation of the example. As in the $1^{\text {st }}$ example, we will also, explain how each connection of the attachments is done, and talk about which Raspberry Pi pins the wires are attached to.

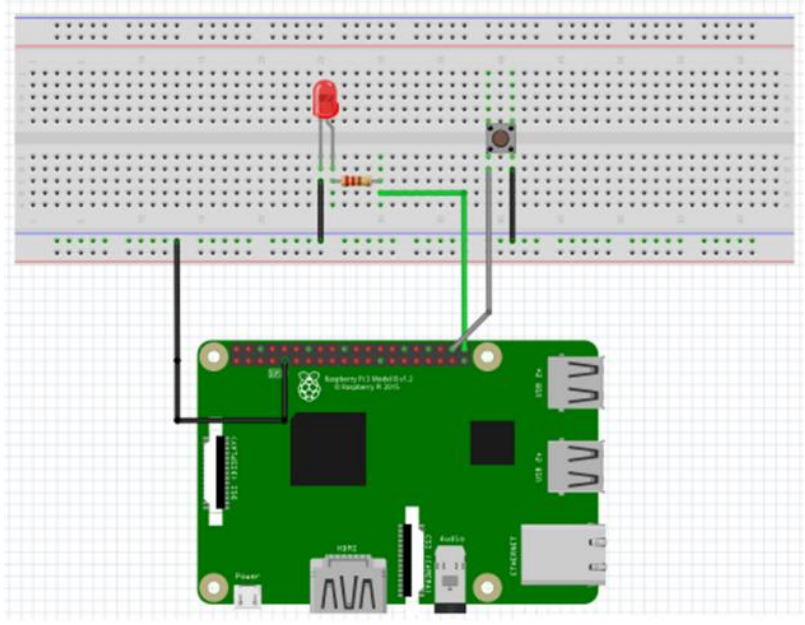

Fig.5: The second application wirings

In the $3^{\text {rd }}$ example, we will discuss two more attachments, apart from the familiar ones (Raspberry Pi, Breadboard, resistor, connection wires). These new attachments are the potentiometer and the MCP3008. We will discuss the function of each attachments, the way each MCP3008 pin works, as well as which of its pins are connected to the Raspberry Pi, and which of them to the rest of the attachments. We will, also, see the circuit that needs to be implemented, the circuit through fritzing, the electrical design and the code. We will explain how each connection of the attachments is done, and talk about which Raspberry Pi pins the wires are attached to.

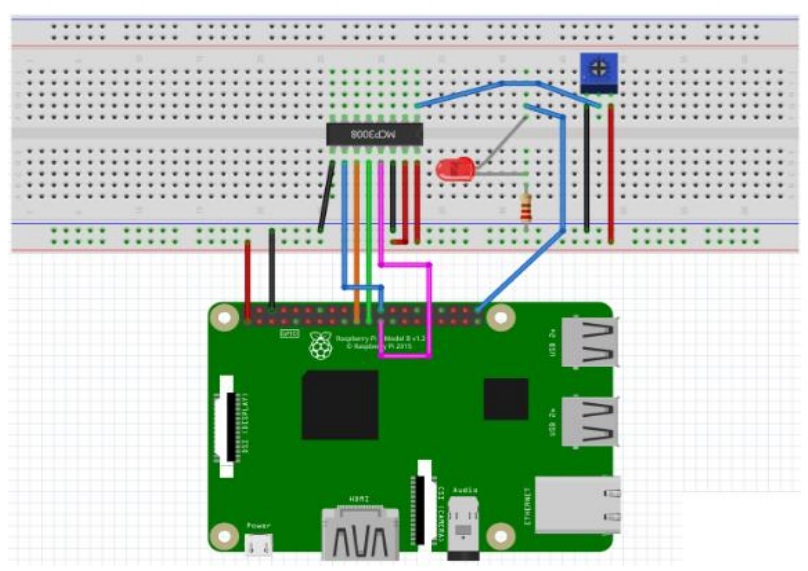

Fig.6: The third application wirings

In the $4^{\text {th }}$ example, we will study closely the following attachments: a Raspberry Pi 3 Model B, a Breadboard, 4 butteries AAA, an L293D, a DC Motor and connection wires. As it appears, there are two new attachments, the L293D and the DC Motor. For this reason, before we move on to the example, we will refer to some basic characteristics of each one of them. First of all, however, we will see which L293D pins are connected to the attachments and which of them to the Raspberry Pi. As soon as this is completed, we will see the circuit through fritzing, the electrical design and the code. We will explain how each connection of attachments is done, and talk about which Raspberry Pi pins the wires are attached to. 


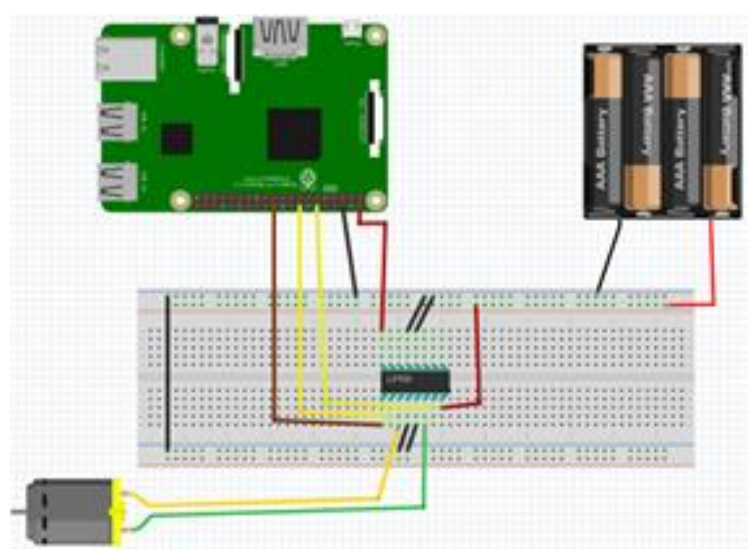

Fig.6: The fourth application wirings

In the $5^{\text {th }}$ example, we will come across the attachments we will work with: a Raspberry Pi 3 Model B, a breadboard, 2 resistors and HC-SR04 wires for the connection. We will look more closely at the HC-SR04 and, as in our other examples; we will see the circuit which needs to be implemented, the circuit through fritzing, the electrical design and the code. We will explain how each connection of the attachments is done, and talk about which Raspberry Pi pins the wires are attached to.

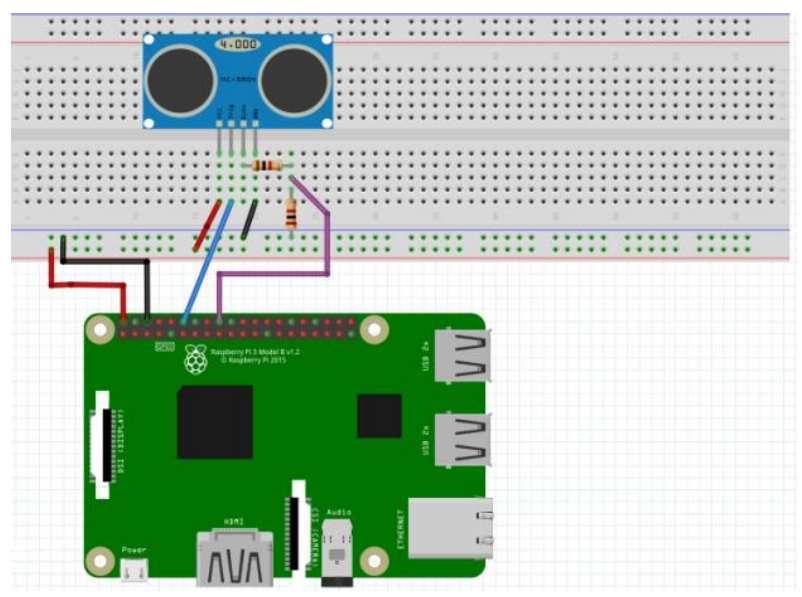

Fig.8: The fifth application wirings

In the $6^{\text {th }}$ and last example, we will see the basic attachments needed for the actualization of the project: a Raspberry Pi 3 Model B, a breadboard, a servo-motor and butteries AAA. We will come across the circuit through fritzing, the electrical design and the code. We will explain how each connection of the attachments is done, and talks about which Raspberry Pi pin the wires are attached to.

\section{CONCLUSIONS}

In this project we tried to present an elementary guide of this specific microcontroller via 5 different examples. As stated in the Intoduction, more information and a more detailed manual of the operation of the microcontroller is available for free upon request.

\section{ACKNOWLEGDEMENTS}

All authors would like to express their gratitude to the Piraeus University of Applied Sciences for providing the required data and funding in order to undertake and complete this research project as part of "Industrial Automation" Postgraduate
Program of Studies.

\section{REFERENCES}

[1] https://www.raspberrypi.org/products/model-a/

[2] https://www.raspberrypi.org/products/model-a-plus/

[3] https://www.raspberrypi.org/products/model-b/

[4] https://www.raspberrypi.org/products/model-b-plus/

[5] https://www.raspberrypi.org/products/raspberry-pi-2model-b/

[6] https://www.raspberrypi.org/products/pi-zero/

[7] https://www.raspberrypi.org/products/raspberry-pi-3model-b/

[8] https://www.elprocus.com/difference-between-arduinoand-raspberry-pi/

[9] https://www.raspberrypi.org/downloads/noobs/

[10] https://sourceforge.net/projects/win32diskimager/

[11] http://www.advanced-ip-scanner.com/gr/

[12] https://www.chiark.greenend.org.uk/ sgtatham/putty/late st.html

[13] https://en.wikipedia.org/wiki/Secure_Shell

[14] http://www.circuitbasics.com/raspberry-pi-basics-setupwithout-monitor-keyboard-headless-mode/

[15] https://learning.raspberrypi.org/en/projects/physicalcomputing

[16] https://learning.raspberrypi.org/en/projects/physicalcomputing

[17] https://learning.raspberrypi.org/en/projects/physicalcomputing

[18] https://learning.raspberrypi.org/en/projects/physicalcomputing

[19] https://learn.adafruit.com/raspberry-pi-analog-to-digitalconverters/mcp3008

[20] http://www.raspberrypi-spy.co.uk/2014/08/enabling-thespi-interface-on-the-raspberry-pi/

[21] https://www.raspberrypi.org/learning/physicalcomputing-with-python/analogue/

[22] https://www.engineersgarage.com/electroniccomponents/1293d-motor-driver-ic

[23] http://randomnerdtutorials.com/complete-guide-forultrasonic-sensor-hc-sr04/

[24] http://www.micropik.com/PDF/HCSR04.pdf

[25] https://www.modmypi.com/blog/hc-sr04-ultrasonicrange-sensor-on-the-raspberry-pi

[26] http://www.seattlerobotics.org/guide/servos.html

[27] http://www.jameco.com/jameco/workshop/howitworks/h ow-servo-motors-work.html

[28] http://www.toptechboy.com/raspberry-pi/raspberry-pilesson-28-controlling-a-servo-on-raspberry-pi-withpython/ 